\title{
On Designing Time-Censored Step-Stress Life Test for the Burr Type-XII Distribution
}

\author{
Ali A. Ismail ${ }^{\mathrm{a}, 1}$ and K. Al-Habardi ${ }^{\mathrm{b}}$ \\ ${ }^{a}$ Cairo University, Faculty of Economics \& Political Science, Department of Statistics, Giza 12613, \\ Egypt \\ b King Saud University, College of Science, Department of Statistics and Operations Research, \\ Riyadh 11451, Saudi Arabia \\ 1 aismail100@yahoo.com (Ali A. Ismail)
}

УДК 539.4

\section{Оптимальное планирование частично ускоренных ресурсных испытаний с цензурированием по времени для распределения Бурра XII типа}

\author{
Али А. Исмаил ${ }^{a}$, Х. Аль-Хабардиб

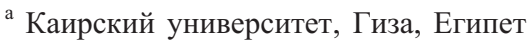 \\ ${ }^{\sigma}$ Университет им. короля Сауда, Эр-Рияд, Саудовская Аравия \\ Предлагается оптимальное планирование частично ускоренных ресурсных испытаний с иензу- \\ рированием по времени для распределения Бурра XII типа. В рамках метода максимальной \\ вероятности получены точечные и интервальные оценки параметров модели. Выполнена \\ оптимизация частично ускоренных ресурсных испытаний с иензурированием по времени. В \\ качестве критерия оптимальности предложена минимизаџия обобщенной асимптотической \\ вариации показателей максимальной вероятности параметров модели. Для опробования \\ методики проведено численное моделирование ряда тестовых задач.
}

Ключевые слова: статистический вывод, оптимальный план испытаний, частично ускоренные ресурсные испытания, максимальная вероятность, распределение Бурра XII типа.

\section{Not ation}

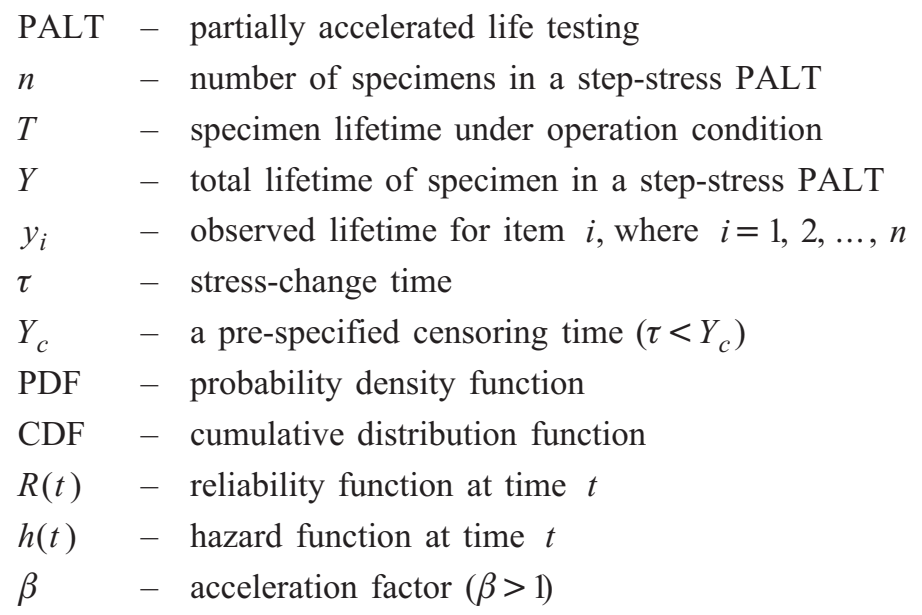


$c, k \quad-$ parameters of the Burr type XII distribution

$n_{u}, n_{a}-$ number of failed items under normal and accelerated conditions, respectively

$y_{(1)} \leq \ldots \leq y_{\left(n_{u}\right)} \leq \tau \leq y_{\left(n_{u}+1\right)} \leq \ldots \leq y_{\left(n_{u}+n_{a}\right)} \leq Y_{c}-$ ordered failure times in a step-stress PALT

Introduction. Generally, the growing need for enhanced reliability increased the application of accelerated test procedures, in order to get important degradation and other information concerning the reliability of products. Such tests aim to offer the accelerated estimation of the product performance.

In practice, the investigation of PALT introduces various essential points. Three types of stresses can be applied to perform such PALT, namely: progressive-stress, step-stress, and constant-stress. The main focus of this research is on the tests of step-stress, which depend on utilizing two levels of stress and can be used in several manners. These tests in turn are divided into two types: failure-step stress and time-step stress. Step-stress scheme allows the stress setting of a unit to be changed upon the occurrence of a fixed number of failures or at pre-specified time. The former is called a failure-step stress test and the latter a time-step stress test which is considered in this paper. As well, optimum test plans are developed in this research to investigate the time step-stress PALT with the assumption that the specimen lifetime follows the Burr type XII distribution.

According to the literature, Degroot and Goel [1] investigated the PALT assuming type-I censoring. The Bayesian approach with various loss functions has been applied to estimate the acceleration parameter and exponential distribution parameters. Bai et al. [2] have discussed the estimation problem for specimens having log-normally distributed lives. Ahmad and Islam [3] have developed optimal accelerated life test designs for the Burr type XII distribution under periodic inspection and type-I censoring. Abdel-Ghaly et al. [4] have studied both the estimation and the optimal design problems assuming that the specimens' lifetimes follow Pareto distribution using type-I censored data. Aly and Ismail [5] have focused on the design problem under step-stress PALT considering Weibull distribution. Abd-Elfattah et al. [6] have studied only the estimation problem of step-stress PALT for the Burr type XII distribution using type-I censoring.

Ismail [7] has presented optimal test plans for time-step PALT assuming a failurecensoring Weibull model. Ismail [8] has studied the optimal design of constant-stress partially accelerated life tests under time-censoring for specimens with lifetimes have Weibull distribution. Ismail [9] has considered optimum PALT plans with progressively type-I interval censored data from Weibull distribution. Ismail [10] has developed optimum failure-censored step-stress life test plans for the Lomax distribution. Recently, Ismail [11] has developed optimum step-stress life tests for the generalized Rayleigh distribution under progressive type-II censoring with binomial removals.

The current study is an extension of the work of Abd-Elfattah et al. [6]. The remaining sections of this paper can be organized as follows. The first section presents the used distribution and the test method. The second section offers the maximum likelihood approach to find both point and interval estimations of the model parameters. The third section considers the developing of optimum PALT plans. The fourth section introduces simulation studies to demonstrate the theoretical aspects.

1. Burr Type XII Distribution as a Lifetime Model. Burr (1942) introduced the distribution in (1) for the first time as a lifetime distribution. In following years, it had been investigated by several researchers due to its broad applications in various areas, mainly in the modeling of failure time and reliability. The PDF, CDF, $R(t)$, and $h(t)$ of the distribution considering the Burr type XII distribution with parameters $(c, k)$ can be expressed as follows, respectively: 


$$
\begin{gathered}
f(t)=c k t^{(c-1)}\left(1+t^{c}\right)^{-(k+1)} t>0, \quad c, k>0, \\
F(t)=1-\left(1+t^{c}\right)^{-(k)}, \quad t>0, \\
R(t)=\left(1+t^{c}\right)^{-(k)}, \quad t>0, \\
h(t)=\frac{c k t^{(c-1)}}{1+t^{c}} .
\end{gathered}
$$

1.1. The Test Method. The test procedure and some basic assumptions for the step-stress PALT are given below:

1.1.1. Test Procedure. The procedure of the experiment is made by initially placing $n$ units on design (normal) stress and run until time $\tau$ at which the stress is changed to a higher one. The test is continued until the censoring time is observed.

1.1.2. Assumptions. The total lifetime $Y$ of an item is as follows:

$$
Y=\left\{\begin{array}{lll}
T & \text { if } & T \leq \tau \\
\tau+\beta^{-1}(T-\tau) & \text { if } & T>\tau
\end{array}\right.
$$

where $T$ is the lifetime of an item at normal condition and $\beta$ is the acceleration factor.

The failure times $y_{i}(i=1, \ldots, n)$ are independent and identically distributed (i.i.d.) random variables (r.v.).

2. Point and Interval Maximum Likelihood Estimations. This Section studies the process of obtaining the maximum likelihood estimates (MLE) of the parameters $(c, k)$ and $\beta$.

2.1. Point Estimation. The observed lifetimes can be expressed as

$$
y_{(1)} \leq \ldots \leq y_{\left(n_{u}\right)} \leq \tau \leq y_{\left(n_{u}+1\right)} \leq \ldots \leq y_{\left(n_{u}+n_{a}\right)} \leq Y_{c} .
$$

The likelihood function of the lifetimes $y_{1}, \ldots, y_{n}$ can be given as follows:

$$
\begin{gathered}
L(y ; \beta, c, k)=\prod_{i=1}^{n}\left\{f_{1}\left(y_{i}\right)\right\}^{\delta_{1 i}}\left\{f_{2}\left(y_{i}\right)\right\}^{\delta_{2 i}}\left\{R\left(Y_{c}\right)\right\}^{\bar{\delta}_{1 i} \bar{\delta}_{2 i}}= \\
\left.=\prod_{i=1}^{n}\left\{c k y^{c-1}\left(1+y_{i}^{c}\right)^{-(k+1)}\right)\right\}^{\delta_{1 i}}\left\{\beta c k\left[\tau+\beta\left(y_{i}-\tau\right)\right]^{c-1} \times\right. \\
\left.\times\left[1+\left(\tau+\beta\left(y_{i}-\tau\right)\right)^{c}\right]^{-(k+1)}\right\}^{\delta_{2 i}}\left\{1+\left[\tau+\beta\left(Y_{c}-\tau\right)\right]^{c}\right\}^{-k \delta_{1 i} \bar{\delta}_{2 i}},
\end{gathered}
$$

where $\bar{\delta}_{1 i}=1-\delta_{1 i}$ and $\bar{\delta}_{2 i}=1-\delta_{2 i}$, and both $\delta_{1 i}$ and $\delta_{2 i}$ can be defined as follows:

$$
\delta_{1 i}=\left\{\begin{array}{ll}
1, & y_{(i)} \leq \tau, \\
0, & \text { otherwise, }
\end{array} \quad i=1,2, \ldots, n,\right.
$$

and

$$
\delta_{2 i}=\left\{\begin{array}{ll}
1, & \tau<y_{(i)} \leq Y_{c}, \\
0, & \text { otherwise },
\end{array} \quad i=1,2, \ldots, n .\right.
$$


Taking the natural logarithm for the expression above of likelihood function, the following formula is obtained

$$
\begin{aligned}
& \ln L=n_{0} \ln c+n_{0} \ln k+(c-1)\left\{\sum_{i=1}^{n} \delta_{1 i} \ln y_{i}+\sum_{i=1}^{n} \delta_{2 i} \ln A\right\}+n_{a} \ln \beta- \\
& -(k+1)\left\{\sum_{i=1}^{n} \delta_{1 i} \ln \left(1+y_{i}^{c}\right)+\sum_{i=1}^{n} \delta_{2 i} \ln \left(1+A^{c}\right)\right\}-k\left(n-n_{0}\right) \ln \left(1+D^{c}\right),
\end{aligned}
$$

where

$$
\begin{gathered}
A=\tau+\beta\left(y_{i}-\tau\right), \quad D=\tau+\beta\left(Y_{c}-\tau\right), \quad \sum_{i=1}^{n} \delta_{1 i}=n_{u}, \quad \sum_{i=1}^{n} \delta_{2 i}=n_{a}, \\
n_{0}=n_{u}+n_{a}, \quad \sum_{i=1}^{n} \bar{\delta}_{1 i} \bar{\delta}_{2 i}=\sum_{i=1}^{n}\left(1-\delta_{1 i}\right)\left(1-\delta_{2 i}\right)=n-n_{u}-n_{a} .
\end{gathered}
$$

The first partial derivatives of the natural logarithm of the likelihood function defined with respect to $\beta$ and $(c, k)$ and are, respectively, given by

$$
\begin{gathered}
\frac{\partial \ln L}{\partial \beta}=\frac{n_{a}}{\beta}+(c-1) \sum_{i=1}^{n} \delta_{2 i}\left(y_{i}-\tau\right) A^{-1}-k c\left(n-n_{0}\right) D^{c-1}\left(Y_{c}-\tau\right)\left(1+D^{c}\right)^{-1}- \\
-(k+1) c \sum_{i=1}^{n} \delta_{2 i} A^{c-1}\left(y_{i}-\tau\right)\left(1+A^{c}\right)^{-1} \\
\frac{\partial \ln L}{\partial c}=\frac{n_{0}}{c}+\sum_{i=1}^{n} \delta_{1 i} \ln y_{i}+\sum_{i=1}^{n} \delta_{2 i} \ln A-k\left(n-n_{0}\right) D^{c} \ln D\left(1+D^{c}\right)^{-1}- \\
-(k+1)\left\{\sum_{i=1}^{n} \delta_{1 i} y_{i}^{c} \ln y_{i}\left(1+y_{i}^{c}\right)^{-1}+\sum_{i=1}^{n} \delta_{2 i} A^{c} \ln A\left(1+A^{c}\right)^{-1}\right\}
\end{gathered}
$$

and

$$
\frac{\partial \ln L}{\partial k}=\frac{n_{0}}{k}-\sum_{i=1}^{n} \delta_{1 i} \ln \left(1+y_{i}^{c}\right)-\sum_{i=1}^{n} \delta_{2 i} \ln \left(1+A^{c}\right)-\left(n-n_{0}\right) \ln \left(1+D^{c}\right) \ldots
$$

By equating Eq. (8) to zero, the MLE of $k$ can be obtained as follows:

$$
\hat{k}=\frac{n_{0}}{a_{1}}
$$

where

$$
a_{1}=\sum_{i=1}^{n} \delta_{1 i} \ln \left(1+y_{i}^{c}\right)+\sum_{i=1}^{n} \delta_{2 i} \ln \left(1+A^{c}\right)+\left(n-n_{0}\right) \ln \left(1+D^{c}\right) .
$$

Substituting Eq. (8) into Eqs. (6) and (7) and equating them to zero, the following two equations are obtained as 


$$
\frac{n_{a}}{\hat{\beta}}+(\hat{c}-1) \sum_{i=1}^{n} \delta_{2 i}\left(y_{i}-\tau\right) A^{-1}-\left(\frac{n_{0}}{a_{1}}+1\right) a_{4}-\frac{n_{0}}{a_{1}} a_{5}=0,
$$

and

$$
\frac{n_{0}}{\hat{c}}+\sum_{i=1}^{n} \delta_{1 i} \ln y_{i}+\sum_{i=1}^{n} \delta_{2 i} \ln A-\left(\frac{n_{0}}{a_{1}}+1\right) a_{2}-\frac{n_{0}}{a_{1}} a_{3}=0
$$

where

$$
\begin{gathered}
a_{2}=\sum_{i=1}^{n} \delta_{1 i} y_{i}^{\hat{c}} \ln y_{i}\left(1+y_{i}^{\hat{c}}\right)^{-1}+\sum_{i=1}^{n} \delta_{2 i} A^{\hat{c}} \ln A\left(1+A^{\hat{c}}\right)^{-1}, \\
a_{3}=\left(n-n_{0}\right) D^{\hat{c}} \ln D\left(1+D^{\hat{c}}\right)^{-1}, \\
a_{4}=\hat{c} \sum_{i=1}^{n} \delta_{2 i} A^{\hat{c}-1}\left(y_{i}-\tau\right)\left(1+A^{\hat{c}}\right)^{-1},
\end{gathered}
$$

and

$$
a_{5}=\left(n-n_{0}\right) \hat{c} D^{\hat{c}-1}\left(Y_{c}-\tau\right)\left(1+D^{\hat{c}}\right)^{-1} .
$$

The MLE of $\beta$ and $c$ can be obtained by solving Eqs. (9) and (10) numerically, while the MLE of $k$ is similarly derived.

Regarding the asymptotic variance-covariance matrix of the MLE of the parameters, it can be approximated by numerically inverting the asymptotic Fisher-information matrix $F$. It is composed of the negative second partial derivatives of the natural logarithm of the likelihood function evaluated at the MLE. Therefore, the asymptotic Fisher-information matrix can be written as

$$
F=\left[\begin{array}{ccc}
-\frac{\partial^{2} \ln L}{\partial^{2} \beta^{2}} & -\frac{\partial^{2} \ln L}{\partial \beta \partial c} & -\frac{\partial^{2} \ln L}{\partial \beta \partial k} \\
-\frac{\partial^{2} \ln L}{\partial c \partial \beta} & -\frac{\partial^{2} \ln L}{\partial^{2} c^{2}} & -\frac{\partial^{2} \ln L}{\partial c \partial k} \\
-\frac{\partial^{2} \ln L}{\partial k \partial \beta} & -\frac{\partial^{2} \ln L}{\partial k \partial c} & -\frac{\partial^{2} \ln L}{\partial^{2} k^{2}}
\end{array}\right] .
$$

Expressions of the elements of such matrix are included in Appendix A, while the determinant of $F$ and its partial derivative with respect to $\tau$ are described in Appendix B.

2.2. Interval Estimates. The estimation of the confidence interval offers a range of values that probably includes the population parameter. For a large sample size, the maximum likelihood estimators are consistent and asymptotically normally distributed. Thus, two-sided approximate confidence limits of the model parameters can be obtained.

3. Optimum Test Plan. In this Section, $\tau$ has to be optimally determined according to a certain optimality criterion. The $D$-optimality criterion is used to achieve this goal. Thus, $\tau$ will be obtained such that the following generalized asymptotic variance (GAV) of the MLE of the model parameters is minimized:

$$
G A V(\hat{\beta}, \hat{c}, \hat{k})=\frac{1}{|F|} .
$$

Practically, the optimum test plans are important for improving the level of precision in parameter estimation and thus improving the quality of the statistical inference. 
4. Simulation Studies. The software has been utilized to carry out all simulation studies, in order to demonstrate all theoretical results. Datasets have been generated from the Burr type XII distribution for various combinations of $c, \beta$, and $k$ parameters, such as $(0.5,2,1),(1.5,2,0.5),(1,2,0.5)$, and $(0.4,2,0.5)$ with sample sizes: $20,25,30,40,50$, 75, and 100. 20,000 replications have been made. Tables 1-4 illustrate the estimation results when $Y_{c}=6$ and $\tau=3$. The results listed in these tables illustrate that the estimates are good. As the sample size increases, both MSE and GAV decrease.

$\mathrm{T}$ a b 1 e 1

The ML Estimates, MSE, Variance, and Confidence Bounds for $\left(c, \beta, k, \tau, y_{c}\right)=(0.5,2,1,3,6)$

\begin{tabular}{|c|c|c|c|c|c|c|}
\hline$n$ & Parameters & Estimates & MSE & Variance & $\begin{array}{l}\text { Lower } \\
\text { bound }\end{array}$ & $\begin{array}{l}\text { Upper } \\
\text { bound }\end{array}$ \\
\hline \multirow[t]{3}{*}{20} & $c$ & 0.5557732 & 0.02035213 & 0.0172 & 0.2475 & 0.7623 \\
\hline & $\beta$ & 1.4271272 & 0.75241109 & 0.4242 & 0.7263 & 3.2796 \\
\hline & $k$ & 1.0610387 & 0.07714338 & 0.0734 & 0.4614 & 1.5236 \\
\hline \multirow[t]{3}{*}{25} & $c$ & 0.5507217 & 0.01509156 & 0.0125 & 0.3284 & 0.7661 \\
\hline & $\beta$ & 1.4584154 & 0.67689516 & 0.3836 & 0.7868 & 3.2147 \\
\hline & $k$ & 1.0507991 & 0.06041368 & 0.0578 & 0.4437 & 1.3864 \\
\hline \multirow[t]{3}{*}{30} & $c$ & 0.5481893 & 0.01300302 & 0.0107 & 0.2506 & 0.6557 \\
\hline & $\beta$ & 1.4879681 & 0.61498619 & 0.3528 & 0.6560 & 2.9844 \\
\hline & $k$ & 1.0458090 & 0.05090453 & 0.0488 & 0.7003 & 1.5663 \\
\hline \multirow[t]{3}{*}{40} & $c$ & 0.544637 & 0.01125746 & 0.0093 & 0.2924 & 2.4076 \\
\hline & $\beta$ & 1.545099 & 0.50244379 & 0.2955 & 0.2766 & 0.6697 \\
\hline & $k$ & 1.035826 & 0.04033314 & 0.0390 & 0.7766 & 1.5513 \\
\hline \multirow[t]{3}{*}{50} & $c$ & 0.526066 & 0.007968931 & 0.0073 & 0.3286 & 0.6633 \\
\hline & $\beta$ & 1.583920 & 0.431667997 & 0.2586 & 0.8412 & 2.8345 \\
\hline & $k$ & 1.033535 & 0.031086777 & 0.0210 & 0.8434 & 1.5219 \\
\hline \multirow[t]{3}{*}{75} & $c$ & 0.5139464 & 0.003147672 & 0.0030 & 0.4811 & 0.6941 \\
\hline & $\beta$ & 1.6458030 & 0.319756411 & 0.1943 & 0.2585 & 1.9864 \\
\hline & $k$ & 1.0199438 & 0.015473287 & 0.0151 & 1.0417 & 1.5231 \\
\hline \multirow[t]{3}{*}{100} & $c$ & 0.5139546 & 0.002524753 & 0.0023 & 0.4823 & 0.6715 \\
\hline & $\beta$ & 1.6854450 & 0.260684144 & 0.1617 & 1.2118 & 2.7883 \\
\hline & $k$ & 1.0184303 & 0.012412655 & 0.0121 & 0.7023 & 1.1331 \\
\hline
\end{tabular}

$\mathrm{T}$ a $\mathrm{b} 1$ e 2

The ML Estimates, MSE, Variance, and Confidence Bounds for $\left(c, \beta, k, \tau, y_{c}\right)=(1.5,2,0.5,3,6)$

\begin{tabular}{|c|c|c|c|c|c|c|}
\hline \hline$n$ & Parameters & Estimates & MSE & Variance & $\begin{array}{c}\text { Lower } \\
\text { bound }\end{array}$ & $\begin{array}{c}\text { Upper } \\
\text { bound }\end{array}$ \\
\hline 1 & 2 & 3 & 4 & 5 & 6 & 7 \\
\hline 20 & $c$ & 1.741682 & 0.7391755 & 0.6808 & 0.0774 & 3.3118 \\
& $\beta$ & 1.608963 & 0.5379832 & 0.3851 & 0.9766 & 3.4091 \\
& $k$ & 0.520448 & 0.0317910 & 0.0314 & 0.0152 & 0.7095 \\
\hline
\end{tabular}


On Designing Time Censored Step-Stress Life Test ...

\begin{tabular}{|c|c|c|c|c|c|c|}
\hline \multicolumn{6}{|c|}{} & \multicolumn{3}{c|}{ Continued Table 2 } \\
\hline \multirow{2}{*}{25} & 2 & 3 & 4 & 5 & 6 & 7 \\
\hline & $c$ & 1.6877987 & 0.2861424 & 0.2509 & 0.5787 & 2.5422 \\
& $\beta$ & 1.6302179 & 0.4702335 & 0.3335 & 0.9434 & 3.2072 \\
\multirow{2}{*}{30} & $k$ & 0.5107501 & 0.0200354 & 0.0199 & 0.1988 & 0.7521 \\
& $c$ & 1.6618421 & 0.2368807 & 0.2107 & 0.3893 & 2.1886 \\
& $\beta$ & 1.6466910 & 0.4200757 & 0.2953 & 0.9528 & 3.0829 \\
& $k$ & 0.5117153 & 0.0165582 & 0.0164 & 0.4659 & 0.9682 \\
\hline \multirow{2}{*}{40} & $c$ & 1.6095242 & 0.11568776 & 0.0130 & 1.2907 & 2.5553 \\
& $\beta$ & 1.6811829 & 0.34308219 & 0.2414 & 1.2237 & 3.1499 \\
& $k$ & 0.5193714 & 0.01339332 & 0.0130 & 0.1014 & 0.5487 \\
\hline \multirow{2}{*}{50} & $c$ & 1.5846642 & 0.08540755 & 0.0782 & 0.8822 & 1.9787 \\
& $\beta$ & 1.7009128 & 0.29636983 & 0.2069 & 0.1910 & 1.9742 \\
& $k$ & 0.5237981 & 0.01221912 & 0.0117 & 0.3935 & 0.8167 \\
\hline \multirow{2}{*}{75} & $c$ & 1.5613964 & 0.052918561 & 0.0492 & 1.2555 & 2.1246 \\
& $\beta$ & 1.7450707 & 0.216361428 & 0.1514 & 0.8074 & 2.1333 \\
& $k$ & 0.5026775 & 0.007078869 & 0.0071 & 0.3710 & 0.7007 \\
\hline \multirow{2}{*}{100} & $c$ & 1.5510198 & 0.038379326 & 0.0358 & 1.1372 & 1.8787 \\
& $\beta$ & 1.7738888 & 0.171096304 & 0.1110 & 1.3647 & 2.7225 \\
& $k$ & 0.5012262 & 0.004537174 & 0.0045 & 0.3754 & 0.6394 \\
\hline
\end{tabular}

$\mathrm{T}$ a b 1 e 3

The ML Estimates, MSE, Variance, and Confidence Bounds

for $\left(c, \beta, k, \tau, y_{c}\right)=(1,2,0.5,3,6)$

\begin{tabular}{|c|c|c|c|c|c|c||}
\hline$n$ & Parameters & Estimates & MSE & Variance & $\begin{array}{c}\text { Lower } \\
\text { bound }\end{array}$ & $\begin{array}{c}\text { Upper } \\
\text { bound }\end{array}$ \\
\hline 1 & 2 & 3 & 4 & 5 & 6 & 7 \\
\hline 20 & $c$ & 1.145478 & 0.16271387 & 0.15868997 & 0.7950 & 3.3797 \\
& $\beta$ & 1.448258 & 0.61302938 & 0.38801491 & 0.1901 & 1.6590 \\
& $k$ & 0.525764 & 0.02171092 & 0.02108904 & 0.1915 & 0.7893 \\
\hline \multirow{2}{*}{25} & $c$ & 1.1316049 & 0.17454634 & 0.1572 & 0.1330 & 1.6874 \\
& $\beta$ & 1.5397050 & 0.55128330 & 0.3394 & 0.9181 & 3.2019 \\
& $k$ & 0.518222 & 0.02067407 & 0.0203 & 0.3183 & 0.8774 \\
\hline \multirow{2}{*}{30} & $c$ & 1.1106032 & 0.11717875 & 0.1041 & 0.5975 & 1.8674 \\
& $\beta$ & 1.5771881 & 0.47564104 & 0.2969 & 0.2393 & 2.3752 \\
& $k$ & 0.5177207 & 0.01771915 & 0.0174 & 0.3313 & 0.8486 \\
\hline \multirow{2}{*}{40} & $c$ & 1.0847688 & 0.05900223 & 0.0518 & 0.7065 & 1.5988 \\
& $\beta$ & 1.6141020 & 0.39836887 & 0.2495 & 0.6834 & 2.6413 \\
& $k$ & 0.5220639 & 0.01461399 & 0.0141 & 0.3043 & 0.7703 \\
\hline \multirow{2}{*}{50} & $c$ & 1.0667929 & 0.04449652 & 0.0400 & 0.8202 & 1.7045 \\
& $\beta$ & 1.6493595 & 0.33485933 & 0.2119 & 0.5055 & 2.3101 \\
& $k$ & 0.5241423 & 0.01317061 & 0.0126 & 0.1743 & 0.6141 \\
\hline
\end{tabular}


Continued Table 3

\begin{tabular}{|c|c|c|c|c|c|c||}
\hline 1 & 2 & 3 & 4 & 5 & 6 & 7 \\
\hline 75 & $c$ & 1.0419664 & 0.022311414 & 0.0206 & 1.0333 & 1.5953 \\
& $\beta$ & 1.6962190 & 0.252072456 & 0.1598 & 0.9461 & 2.5131 \\
& $k$ & 0.5028481 & 0.005919429 & 0.0059 & 0.1957 & 0.4971 \\
\hline \multirow{2}{*}{100} & $c$ & 1.0313848 & 0.015573608 & 0.0146 & 0.8892 & 1.3627 \\
& $\beta$ & 1.7333837 & 0.198241968 & 0.1272 & 0.5596 & 1.9574 \\
& $k$ & 0.5051782 & 0.004335673 & 0.0043 & 0.4292 & 0.6865 \\
\hline
\end{tabular}

$\mathrm{T}$ a b 1 e 4

The ML Estimates, MSE, Variance, and Confidence Bounds for $\left(c, \beta, k, \tau, y_{c}\right)=(0.4,2,0.5,3,6)$

\begin{tabular}{|c|c|c|c|c|c|c||}
\hline$n$ & Parameters & Estimates & MSE & Variance & $\begin{array}{c}\text { Lower } \\
\text { bound }\end{array}$ & $\begin{array}{c}\text { Upper } \\
\text { bound }\end{array}$ \\
\hline 20 & $c$ & 0.4983496 & 0.25866466 & 0.2490 & 0.1901 & 0.7462 \\
& $\beta$ & 1.3373836 & 0.92303761 & 0.4840 & 0.6542 & 3.3505 \\
& $k$ & 0.5213906 & 0.03163609 & 0.0312 & 0.0705 & 0.7463 \\
\hline 25 & $c$ & 0.4680883 & 0.05913880 & 0.0545 & 0.1903 & 0.6681 \\
& $\beta$ & 1.3762620 & 0.83451385 & 0.4455 & 0.6769 & 3.3258 \\
& $k$ & 0.5202933 & 0.02627366 & 0.0259 & 0.2276 & 0.7189 \\
\hline \multirow{2}{*}{30} & $c$ & 0.4425822 & 0.02958456 & 0.0278 & 0.1727 & 0.5842 \\
& $\beta$ & 1.4156463 & 0.74620001 & 0.4048 & 0.7366 & 3.2637 \\
& $k$ & 0.5137014 & 0.01832341 & 0.0181 & 0.1652 & 0.6912 \\
\hline \multirow{2}{*}{40} & $c$ & 0.4256365 & 0.008456827 & 0.0078 & 0.2101 & 0.5649 \\
& $\beta$ & 1.4811799 & 0.612796371 & 0.3436 & 0.9004 & 3.1005 \\
& $k$ & 0.5116403 & 0.011437146 & 0.0113 & 0.2836 & 0.6737 \\
\hline \multirow{2}{*}{50} & $c$ & 0.4243705 & 0.006428742 & 0.0058 & 0.3749 & 0.6621 \\
& $\beta$ & 1.5315188 & 0.518581400 & 0.2991 & 0.6086 & 2.7428 \\
& $k$ & 0.5093500 & 0.009389015 & 0.0093 & 0.2319 & 0.6026 \\
\hline 75 & $c$ & 0.4222316 & 0.004573514 & 0.0041 & 0.3768 & 0.6346 \\
& $\beta$ & 1.5983929 & 0.396886760 & 0.2356 & 1.0306 & 2.9693 \\
& $k$ & 0.5083808 & 0.007263108 & 0.0072 & 0.2836 & 0.6411 \\
\hline \multirow{2}{*}{100} & $c$ & 0.4169281 & 0.004095201 & 0.0038 & 0.2506 & 0.4948 \\
& $\beta$ & 1.6402711 & 0.325215087 & 0.1958 & 1.1094 & 2.8906 \\
& $k$ & 0.5042987 & 0.006526954 & 0.0065 & 0.3223 & 0.6336 \\
\hline
\end{tabular}

Tables 5-8 illustrate the optimum stress-change time $\tau^{*}$, which ensures the minimal value of GAV of the MLE for the model parameters under the operation conditions. Accordingly, both $n_{u}^{*}$ and $n_{a}^{*}$, the expected optimal numbers of failed items under normal and accelerated conditions, respectively, have been obtained. The optimal design of the life test can achieve more savings in time and cost, and more accurate estimates of the model parameters can be obtained.

Conclusions. In this paper, the optimal design problem of the step-stress PALT model has been discussed under the Burr type-XII distribution as a lifetime distribution of the test units assuming the type-I censoring. The test specimen was first run under the operation 
On Designing Time Censored Step-Stress Life Test ...

T a b 1 e 5

The Results of the Optimal Design of the Life Test for $y_{c}=6$ and $(c, \beta, k)=(0.5,2,1)$

\begin{tabular}{||c|c|c|c|c||}
\hline$n$ & $\tau^{*}$ & $n_{u}^{*}=n P_{u}$ & $n_{a}^{*}=n P_{a}$ & Optimal GAV \\
\hline 20 & 3.905571 & 14 & 4 & 0.00042 \\
\hline 25 & 3.972806 & 17 & 5 & 0.00023 \\
\hline 30 & 3.843423 & 21 & 6 & 0.00022 \\
\hline 40 & 3.722781 & 27 & 11 & 0.00012 \\
\hline 50 & 3.510012 & 34 & 18 & 0.00004 \\
\hline 75 & 3.350125 & 49 & 24 & 0.00002 \\
\hline 100 & 3.264495 & 65 & 9 & 0.00001 \\
\hline
\end{tabular}

T a b 1 e 6

The Results of the Optimal Design of the Life Test for $y_{c}=6$ and $(c, \beta, k)=(1.5,2,0.5)$

\begin{tabular}{||c|c|c|c|c||}
\hline$n$ & $\tau^{*}$ & $n_{u}^{*}=n P_{u}$ & $n_{a}^{*}=n P_{a}$ & Optimal GAV \\
\hline 20 & 3.877339 & 14 & 4 & 0.00185 \\
\hline 25 & 3.541986 & 17 & 7 & 0.00046 \\
\hline 30 & 3.456714 & 20 & 9 & 0.00020 \\
\hline 40 & 3.494583 & 27 & 11 & 0.00012 \\
\hline 50 & 3.53408 & 34 & 20 & 0.00011 \\
\hline 75 & 3.220774 & 48 & 28 & 0.00003 \\
\hline 100 & 3.123217 & 62 & 20002 \\
\hline
\end{tabular}

T a b 1 e 7

The Results of the Optimal Design of the Life Test for $y_{c}=6$ and $(c, \beta, k)=(1,2,0.5)$

\begin{tabular}{|c|c|c|c|c||}
\hline$n$ & $\tau^{*}$ & $n_{u}^{*}=n P_{u}$ & $n_{a}^{*}=n P_{a}$ & Optimal GAV \\
\hline 20 & 3.8834 & 11 & 4 & 0.00108 \\
\hline 25 & 3.6262 & 14 & 7 & 0.00044 \\
\hline 30 & 3.7343 & 17 & 10 & 0.00024 \\
\hline 40 & 3.4431 & 22 & 13 & 0.00009 \\
\hline 50 & 3.4672 & 27 & 20 & 0.00003 \\
\hline 75 & 3.3252 & 40 & 28 & 0.00002 \\
\hline 100 & 3.1769 & 52 & 0.00001 \\
\hline
\end{tabular}


$\mathrm{T}$ a b 1 e 8

The Results of the Optimal Design of the Life Test for $y_{c}=6$ and $(c, \beta, k)=(0.4,2,0.5)$

\begin{tabular}{|c|c|c|c|c||}
\hline$n$ & $\tau^{*}$ & $n_{u}^{*}=n P_{u}$ & $n_{a}^{*}=n P_{a}$ & Optimal GAV \\
\hline 20 & 4.0838 & 9 & 4 & 0.00061 \\
\hline 25 & 3.8823 & 10 & 6 & 0.00015 \\
\hline 30 & 3.8470 & 12 & 7 & 0.00012 \\
\hline 40 & 4.1866 & 17 & 9 & 0.00011 \\
\hline 50 & 3.6482 & 20 & 12 & 0.00003 \\
\hline 75 & 3.7362 & 30 & 25 & 0.00001 \\
\hline 100 & 3.4378 & 39 & & 0.000006 \\
\hline
\end{tabular}

condition, and if it did not fail until a pre-specified time, the stress was raised, that is, it was tested under the accelerated condition until its failure or the test termination.

The maximum likelihood estimates of the model parameters have been obtained. In addition, optimum test plans have been developed such that the GAV of the MLE of the model parameters was minimized. These plans allow one to determine the optimal value of the switching time $\tau^{*}$ that minimizes the GAV. Accordingly, the quality of the statistical inference can be improved.

\section{Appendix A}

Derivation of the second-order partial derivatives:

$$
\begin{aligned}
& \frac{\partial^{2} \ln L}{\partial^{2} \beta^{2}}=-\frac{n_{a}}{\beta^{2}}-(c-1) \sum_{i=1}^{n} \delta_{2 i}\left(y_{i}-\tau\right) \frac{\left(y_{i}-\tau\right)}{\left(\tau+\beta\left(y_{i}-\tau\right)\right)^{2}}- \\
& -(k+1) c \sum_{i=1}^{n}\left(y_{i}-\tau\right) \delta_{2 i}\left[\left(y_{i}-\tau\right)(c-1) A^{c-2}\left(1+A^{c}\right)^{-1}-\right. \\
& \left.-\left(y_{i}-\tau\right) c A^{2(c-1)}\left(1+A^{c}\right)^{-2}\right]-k c\left(n-n_{0}\right)\left(Y_{c}-\tau\right)\left[(c-1)\left(Y_{c}-\tau\right) D^{c-2}\left(1+D^{c}\right)^{-1}-\right. \\
& \left.-\left(Y_{c}-\tau\right) c D^{2(c-1)}\left(1+D^{c}\right)^{-2}\right]=-\frac{n_{a}}{\beta^{2}}-(c-1) \sum_{i=1}^{n} \delta_{2 i}\left(y_{i}-\tau\right)^{2} A^{-2}- \\
& -(k+1) c \sum_{i=1}^{n}\left(y_{i}-\tau\right)^{2} \delta_{2 i}\left[(c-1) A^{c-2}\left(1+A^{c}\right)^{-1}-c A^{2(c-1)}\left(1+A^{c}\right)^{-2}\right]- \\
& -k c\left(n-n_{0}\right)\left(Y_{c}-\tau\right)^{2}\left[(c-1) D^{c-2}\left(1+D^{c}\right)^{-1}-c D^{2(c-1)}\left(1+D^{c}\right)^{-2}\right], \\
& \frac{\partial^{2} \ln L}{\partial \beta \partial c}=\sum_{i=1}^{n} \delta_{2 i}\left(y_{i}-\tau\right) A^{-1}-k\left(Y_{c}-\tau\right)\left(n-n_{0}\right) \times \\
& \times\left[D^{c-1}\left(1+D^{c}\right)^{-1}+c\left(1+D^{c}\right)^{-1} D^{c-1} \ln D-c D^{c-1}\left(1+D^{c}\right)^{-2} D^{c} \ln D\right]-
\end{aligned}
$$




$$
\begin{gathered}
-(k+1) \sum_{i=1}^{n} \delta_{2 i}\left(y_{i}-\tau\right)\left[A^{c-1}\left(1+A^{c}\right)^{-1}+c\left(1+A^{c}\right)^{-1} A^{c-1} \ln A-\right. \\
\left.-c A^{c-1}\left(1+A^{c}\right)^{-2} A^{c} \ln A\right], \\
\frac{\partial^{2} \ln L}{\partial \beta \partial k}=-c \sum_{i=1}^{n} \delta_{2 l}\left(y_{i}-\tau\right) A^{c-1}\left(1+A^{c}\right)^{-1}-\left(Y_{c}-\tau\right)\left(n-n_{0}\right) c D^{c-1}\left(1+D^{c}\right)^{-1}, \\
\frac{\partial^{2} \ln L}{\partial c^{2}}=-\frac{n_{0}}{c^{2}}-k\left(n-n_{0}\right) \ln D\left[\left(1+D^{c}\right)^{-1} D^{c} \ln D-\left(1+D^{c}\right)^{-2} D^{2 c} \ln D\right]- \\
-(k+1)\left[\sum_{i=1}^{n} \delta_{1 i} \ln y_{i}\left\{\left(1+y_{i}^{c}\right)^{-1} y_{i}^{c} \ln y_{i}-y_{i}^{2 c}\left(1+y_{i}^{c}\right)^{-2} \ln y_{i}\right\}\right]- \\
-(k+1)\left[\sum_{i=1}^{n} \delta_{2 i} \ln A\left\{\left(1+A^{c}\right)^{-1} A^{c} \ln A-A^{2 c}\left(1+A^{c}\right)^{-2} \ln A\right\}\right] \\
\frac{\partial^{2} \ln L}{\partial c \partial k}=-\left(n-n_{0}\right)\left(1+D^{c}\right)^{-1} D_{1 i}^{c} \ln y_{i}\left\{\left(1+y_{i}^{c}\right)^{-1} y_{i}^{c} \ln y_{i}\right\}-\sum_{i=1}^{n} \delta_{2 i} \ln A\left(1+A^{c}\right)^{-1} A^{c} \ln A- \\
-(n-1)
\end{gathered}
$$

and

$$
\frac{\partial^{2} \ln L}{\partial k^{2}}=-\frac{n_{0}}{k^{2}} .
$$

\section{Appendix B}

The determinant of $F$ and its partial derivative with respect to $\tau$. The determinant of $F$ can be written as

$$
|F|=f_{11}\left(f_{22} f_{33}-f_{23}^{2}\right)-f_{12}\left(f_{12} f_{33}-f_{13} f_{23}\right)+f_{13}\left(f_{12} f_{23}-f_{13} f_{22}\right),
$$

and then

$$
\begin{gathered}
\frac{\partial|F|}{\partial \tau}=f_{11}\left(f_{22}^{\prime} f_{33}+f_{22} f_{33}^{\prime}-2 f_{23} f_{23}^{\prime}\right)+f_{11}^{\prime}\left(f_{22} f_{33}-f_{23}^{2}\right)- \\
-f_{12}\left(f_{12}^{\prime} f_{33}+f_{12} f_{33}^{\prime}-f_{13}^{\prime} f_{23}-f_{13} f_{23}^{\prime}\right)-f_{12}^{\prime}\left(f_{12} f_{33}-f_{13} f_{23}\right)+ \\
+f_{13}\left(f_{12}^{\prime} f_{23}+f_{12} f_{23}^{\prime}-f_{13}^{\prime} f_{22}-f_{13} f_{22}^{\prime}\right)+f_{13}^{\prime}\left(f_{12} f_{23}-f_{13} f_{22}\right)
\end{gathered}
$$

where

$$
\begin{aligned}
& f_{11}^{\prime}=(c-1) \sum_{i=1}^{n} \delta_{2 i}\left[-2\left(y_{i}-\tau\right) A^{-2}-2\left(y_{i}-\tau\right)^{2} A^{-3}(1-\beta)\right]+ \\
& +(k+1) c \sum_{i=1}^{n}(c-1) \delta_{2 i}\left[-2\left(y_{i}-\tau\right) A^{c-2}\left(1+A^{c}\right)^{-1}+\left(y_{i}-\tau\right)^{2} \times\right.
\end{aligned}
$$




$$
\begin{aligned}
& \left.\times(1-\beta)\left((c-2) A^{c-3}\left(1+A^{c}\right)^{-1}-c A^{2 c-3}\left(1+A^{c}\right)^{-2}\right)\right]- \\
& -(k+1) c \sum_{i=1}^{n} c \delta_{2 i}\left[-2\left(y_{i}-\tau\right) A^{2(c-1)}\left(1+A^{c}\right)^{-2}+\left(y_{i}-\tau\right)^{2} 2(1-\beta) \times\right. \\
& \left.\times\left((c-1) A^{2 c-3}\left(1+A^{c}\right)^{-2}-c A^{3(c-1)}\left(1+A^{c}\right)^{-3}\right)\right]+ \\
& +k c\left(n-n_{0}\right)(c-1)\left[-2\left(Y_{c}-\tau\right) D^{c-2}\left(1+D^{c}\right)^{-1}+(1-\beta)\left(Y_{c}-\tau\right)^{2} \times\right. \\
& \left.\times\left((c-2) D^{c-3}\left(1+D^{c}\right)^{-1}-c D^{2 c-3}\left(1+D^{c}\right)^{-2}\right)\right]- \\
& -k c^{2}\left(n-n_{0}\right)\left[-2\left(Y_{c}-\tau\right) D^{2(c-1)}\left(1+D^{c}\right)^{-2}+2\left(Y_{c}-\tau\right)^{2}(1-\beta) \times\right. \\
& \left.\times\left((c-1) D^{2 c-3}\left(1+D^{c}\right)^{-2}-D^{3(c-1)} c\left(1+D^{c}\right)^{-3}\right)\right], \\
& f_{22}^{\prime}=k\left(n-n_{0}\right)(1-\beta)\left[2 D^{c-1}\left(1+D^{c}\right)^{-1} \ln D-c(\ln D)^{2} \times\right. \\
& \left.\times\left(D^{2 c-1}\left(1+D^{c}\right)^{-2}+D^{c-1}\left(1+D^{c}\right)^{-1}\right)\right]- \\
& -k\left(n-n_{0}\right)(1-\beta)\left[D^{2 c-1}\left(1+D^{c}\right)^{-2} 2 \ln D+2 c(1-\beta)(\ln D)^{2} \times\right. \\
& \left.\times\left\{-D^{3 c-1}\left(1+D^{c}\right)^{-3}+D^{2 c-1}\left(1+D^{c}\right)^{-2}\right\}\right]+ \\
& +(k+1)(1-\beta) \sum_{i=1}^{n} \delta_{2 i}\left[2 A^{c-1}\left(1+A^{c}\right)^{-1} \ln A+c(\ln A)^{2} \times\right. \\
& \left.\times\left\{-\left(1+A^{c}\right)^{-2} A^{2 c-1}+\left(1+A^{c}\right)^{-1} A^{c-1}\right\}\right]- \\
& -(k+1)(1-\beta) \sum_{i=1}^{n} \delta_{2 i}\left[2 A^{2 c-1}\left(1+A^{c}\right)^{-2} \ln A+2 c(\ln A)^{2} \times\right. \\
& \times\left\{\left(1+A^{c}\right)^{-2} A^{2 c-1}-\left(1+A^{c}\right)^{-3} A^{3 c-1}\right\}, \\
& f_{33}^{\prime}=0 \text {, } \\
& f_{23}^{\prime}=\sum_{i=1}^{n} \delta_{2 i}(1-\beta)\left[2 A^{c-1}\left(1+A^{c}\right)^{-1} \ln A+c(\ln A)^{2} \times\right. \\
& \left.\times\left(1+A^{c}\right)^{-1} A^{c-1}-\left(1+A^{c}\right)^{-1} A^{2 c-1}\right]+ \\
& +\left(n-n_{0}\right)(1-\beta)\left[-D^{2 c-1}\left(1+D^{c}\right)^{-2} c \ln D+\left(1+D^{c}\right)^{-1} D^{c-1}\{c \ln D+1\}\right], \\
& f_{12}^{\prime}=\sum_{i=1}^{n} \delta_{2 i}\left[A^{-1}+(1-\beta)\left(y_{i}-\tau\right) A^{-2}\right]+k\left(n-n_{0}\right)\left\{(1-\beta)\left(Y_{c}-\tau\right) \times\right.
\end{aligned}
$$




$$
\begin{gathered}
\times\left[D^{c-2}(c-1)\left(1+D^{c}\right)^{-1}-c\left(1+D^{c}\right)^{-2} D^{2(c-1)}\right]-D^{c-1}\left(1+D^{c}\right)^{-1}- \\
-c\left(1+D^{c}\right)^{-1} D^{c-1} \ln D+c\left(Y_{c}-\tau\right)(1-\beta)\left\{-D^{2(c-1)} c\left(1+D^{c}\right)^{-2} \ln D+\right. \\
\left.+D^{c-2}\left(1+D^{c}\right)^{-1}[1+(c-1) \ln D]\right\}+c D^{2 c-1}\left(1+D^{c}\right)^{-2} \ln D-c(1-\beta)\left(Y_{c}-\tau\right) \times \\
\left.\times\left[D^{2 c-2}\left(1+D^{c}\right)^{-2}-2 c\left(1+D^{c}\right)^{-3} D^{3 c-2} \ln D+(2 c-1)\left(1+D^{c}\right)^{-2} D^{2 c-2} \ln D\right]\right\}+ \\
+(k+1) \sum_{i=1}^{n} \delta_{2 i}\left(y_{i}-\tau\right)\left\{( 1 - \beta ) \left[(c-1) A^{c-2}\left(1+A^{c}\right)^{-1}-c A^{2(c-1)}\left(1+A^{c}\right)^{-2}-\right.\right. \\
-c^{2} A^{2(c-1)}\left(1+A^{c}\right)^{-2} \ln A+c A^{c-2}\left(1+A^{c}\right)^{-1}(1+(c-1) \ln A)-c A^{2(c-1)}\left(1+A^{c}\right)^{-2}+ \\
\left.+2 c^{2} A^{3 c-2}\left(1+A^{c}\right)^{-3} \ln A-c(2 c-1) A^{2 c-2}\left(1+A^{c}\right)^{-2} \ln A\right\}- \\
-\left[A^{c-1}\left(1+A^{c}\right)^{-1}+c\left(1+A^{c}\right)^{-1} A^{c-1} \ln A-c A^{2 c-1}\left(1+A^{c}\right)^{-2} \ln A\right], \\
f_{13}^{\prime}=\sum_{i=1}^{n} \delta_{2 i} c\left\{\left(y_{i}-\tau\right)(1-\beta)\left[(c-1) A^{c-2}\left(1+A^{c}\right)^{-1}-c A^{2(c-1)}\left(1+A^{c}\right)^{-2}\right]-\right. \\
\left.-A^{c-1}\left(1+A^{c}\right)^{-1}\right\}+\left(n-n_{0}\right) c\left[( Y _ { c } - \tau ) ( 1 - \beta ) \left[(c-1) D^{c-2}\left(1+D^{c}\right)^{-1}-\right.\right. \\
\left.\left.-c D^{2(c-1)}\left(1+D^{c}\right)^{-2}\right]-D^{c-1}\left(1+D^{c}\right)^{-1}\right] .
\end{gathered}
$$

\section{Резюме}

Пропонується оптимальне планування частково прискорених ресурсних випробувань iз цензуруванням у часі для розподілу Бурра XII типу. У рамках методу максимальної імовірності отримано точкові й інтервальні оцінки параметрів моделі. Виконано оптимізацію частково прискорених ресурсних випробувань із цензуруванням у часі. Як критерій оптимальності пропонується мінімізація узагальненої асимптотичної варіації показників максимальної імовірності параметрів моделі. Для опробування методики проведено чисельне моделювання ряду тестових задач.

1. M. H. Degroot and P. K. Goel, "Bayesian estimation and optimal designs in partially accelerated life testing," Nav. Res. Logist. Q., 26, No. 2, 223-235 (1979).

2. D. S. Bai, S. W. Chung, and Y. R. Chun, "Optimal design of partially accelerated life tests for the lognormal distribution under type I censoring," Reliab. Eng. Syst. Safe., 40, No. 1, 85-92 (1993).

3. N. Ahmad and A. Islam, "Optimal accelerated life test designs for Burr type XII distributions under periodic inspection and type I censoring," Nav. Res. Log., 43, No. 8, 1049-1077 (1996).

4. A. A. Abdel-Ghaly, E. H. El-Khodary, and A. A. Ismail, "Maximum likelihood estimation and optimal design in step partially accelerated life tests for the Pareto distribution with type-I censoring," in: Proc. of the 14th Annual Conf. on Statistics and Computer Modeling in Human and Social Sciences, Cairo University (2002), pp. 1629. 
5. H. M. Aly and A. A. Ismail, "Optimum simple time-step stress plans for partially accelerated life testing with censoring," Far East J. Theor. Stat., 24, No. 2, 175-200 (2008).

6. A. M. Abd-Elfattah, A. S. Hassan, and S. G. Nassr, "Estimation in step-stress partially accelerated life tests for the Burr type XII distribution using type I censoring," Stat. Methodol., 5, No. 6, 502-514 (2008).

7. A. A. Ismail, "On designing step-stress partially accelerated life tests under failurecensoring scheme," P. I. Mech. Eng. O - J. Ris., 227, No. 6, 662-670 (2013).

8. Ali A. Ismail, "On designing constant-stress partially accelerated life tests under time-censoring," Strength Mater., 46, No. 1, 132-139 (2014).

9. A. A. Ismail, "Optimum partially accelerated life test plans with progressively type I interval-censored data," Sequential Anal., 34, No. 2, 135-147 (2015).

10. Ali A. Ismail, "A. A. Ismail, "Optimum failure-censored step-stress life test plans for the Lomax distribution," Strength Mater., 48, No. 3, 437-443 (2016).," Strength Mater., 48, No. 3, 437-443 (2016).

11. Ali A. Ismail, "Planning step-stress life tests for the generalized Rayleigh distribution under progressive type-II censoring with binomial removals," Strength Mater., 49, No. 2, 292-306 (2016). 